\title{
LA CONVENCIÓN DE VIENA SOBRE CONTRATOS DE COMPRAVENTA INTERNACIONAL DE MERCADERÍAS EN EL CONTEXTO DEL MERCOSUR: ¿HERRAMIENTA PARA UNA MAYOR INTEGRACIÓN?
}

A CONVENCÃO DE VIENA SOBRE A COMPRA E VENDA INTERNACIONAL DE MERCADORIAS NO CONTEXTO DO MERCOSUL: FERRAMENTA PARA UMA MAIOR INTEGRAÇÃO?

Pablo Antonio Debuchy Boselli*

Resumen: La reciente adhesión de Brasil a la Convención de las Naciones Unidas sobre Contratos de Compraventa Internacional de Mercaderías (CNUCCIM) representa un importante impulso para la uniformidad del derecho privado en el MERCOSUR. Esta Convención, de amplia aceptación y utilización a nivel mundial, busca contribuir en un nuevo orden económico internacional, sobre la base de la armonía y la igualdad $y$ en aras de la supresión de los obstáculos jurídicos característicos del comercio transfronterizo, objetivo afín con el de la creación de un mercado común. Sin embargo, es una realidad que existen asimetrías entre los países del bloque regional en cuanto a la incorporación de la Convención en la legislación doméstica, así como en la aplicación de sus reglas en la jurisprudencia nacional. El propósito de este trabajo es el de identificar tales asimetrías, y esbozar algunas hipótesis acerca de los motivos de la limitada jurisprudencia sobre la materia en la región, buscando así provocar un estudio más detallado sobre la conveniencia y los medios adecuados para promover el uso de esta herramienta del derecho uniforme en el MERCOSUR.

Resumo: A recente adesão do Brasil à Convenção das Nações Unidas sobre os Contratos de Compra e Venda Internacional de Mercadorias (CISG), representa um grande impulso para uniformidade do direito privado no MERCOSUL. A presente Convenção, com ampla aceitação

* Universidad Católica Nuestra Señora de la Asunción, Paraguay.

E-mail: pablodebuchyb@gmail.com

Recibido: 1/02/2017. Aceptado: 3/03/2017. 
e uso em todo o mundo, busca contribuir para uma nova ordem internacional econômica, com base na harmonia, igualdade e supressão dos obstáculos legais característicos para o comércio transfronteiriço, a meta da criação, objetivo afim com um mercado comum. No entanto, é uma realidade que existem assimetrias entre os países da região sobre a incorporação da Convenção a legislação nacional e na aplicação das suas regras a jurisprudência nacional. O objetivo deste trabalho é identificar as assimetrias, e delinear algumas hipóteses sobre as razões para a limitada jurisprudência sobre CISG na região, para incitar um estudo mais detalhado sobre a conveniência e os meios adequados para promover o uso desta ferramenta de direito uniforme no MERCOSUL.

Palabras clave: CNUCCIM, Compraventa internacional, Derecho uniforme, MERCOSUR

Palavras-chave: CISG, Compra e venda internacional, Direito uniforme, MERCOSUL

\section{INTRODUCCIÓN}

Los países que integran el Mercado Común del Sur (MERCOSUR) comparten una estrecha afinidad jurídica en materia de derecho privado, especialmente en lo que respecta a los principios generales de los contratos comerciales.

Esta debería ser una importante ventaja en relación con otros esfuerzos de integración regional, incluso en relación con la Unión Europea, en la que convergen sistemas jurídicos sustancialmente distintos, máxime atendiendo que el propósito central del MERCOSUR es la libre circulación de bienes, servicios y factores productivos, según el artículo 1 del Tratado de Asunción.

Teniendo como norte este objetivo, la uniformidad del derecho privado de los países miembros del mercado común es el escenario ideal para agilizar las relaciones comerciales, desarrollar jurisprudencia constante y pacífica a nivel regional, y en consecuencia ofrecer mayor predictibilidad y seguridad jurídica.

Sin embargo, y a pesar de estas condiciones favorables, la experiencia de los países latinoamericanos en la unificación del derecho privado ha sido, en líneas generales, decepcionante, especialmente debido al escepticismo jurídico fundado en no pocas ocasiones en el discurso de la protección de la soberanía nacional, que termina por ralentizar -o frustrar definitivamente- los numerosos procesos de integración.

En tiempos en los que toda la atención del MERCOSUR parece estar centrada en la solución de cuestiones eminentemente políticas, representa un interesante impulso para la unificación la adhesión de 
Brasil a la Convención de las Naciones Unidas sobre los Contratos de Compraventa Internacional de Mercaderías (la Convención o la CNUCCIM), instrumento de derecho uniforme por excelencia que regula este tipo de contratación clave para el comercio internacional, y cuya finalidad es prever un régimen moderno y equitativo, contribuyendo así notablemente a dar seguridad jurídica a los intercambios comerciales y a reducir los gastos de las operaciones.

Este trabajo tiene por objeto exponer algunas características generales de la Convención, así como su situación normativa y jurisprudencial en los países del MERCOSUR, para pasar luego a identificar los eventuales motivos de su aún escasa aplicación y, finalmente, formular algunas conclusiones que sirvan para un estudio más acabado acerca de la conveniencia de su promoción y aplicación en el bloque regional.

\section{CONSIDERACIONES GENERALES SOBRE LA CNUCCIM Y SU IMPORTANCIA EN LA CONSOLIDACIÓN DEL DERECHO UNIFORME}

La corriente jurídica conocida como derecho uniforme, de vertiginoso desarrollo desde la segunda mitad del siglo XX, ciertamente tiene características específicas que la distinguen del derecho de la integración en el que campea por esencia la normativa mercosureña, aunque sus objetivos están estrechamente relacionados en última instancia, pudiendo servir el primero como instrumento de fortalecimiento del segundo.

En efecto, el derecho de la integración y el derecho uniforme convergen en la matriz normativa originaria del derecho internacional general. El primero tiene por objetivo primordial regular las conductas de los Estados entre sí, de estos con organismos internacionales, y de estos organismos entre sí, a fin de potenciar la cooperación entre las partes para facilitar la obtención de los fines propuestos, eminentemente económicos en el caso del MERCOSUR.

El segundo tiene su origen, más que en el derecho internacional strictu sensu, en el notable incremento del comercio internacional, siendo su aspiración esencial la uniformidad de reglas, de validez potencialmente universal, para disciplinar una concreta conducta transfronteriza cualquiera sea la nacionalidad de las partes obligadas, el lugar de la prestación o el emplazamiento de la cosa en relación con la cual la conducta debe ser satisfecha.

Un rasgo diferenciador entre ambas disciplinas se observa en su esfera de incidencia. El derecho de la integración, y específicamente el modelo adoptado por el MERCOSUR incluye normas de carácter jurídico-político que pretenden coordinar los esfuerzos de los poderes 
políticos involucrados -i.e. los países miembros, organizados en un bloque intergubernamental - a fin de regular la actividad de los particulares que se desenvuelven en el comercio internacional. El derecho uniforme en cambio tiene un objetivo mucho más limitado y no aspira a inferir en la soberanía económica de los Estados, sino más bien aspira a que dicha soberanía les permita gobernar las concretas relacionados privadas y libres de sus ciudadanos individuales, constituyéndose así en un orden eminentemente jurídico-privado que disciplina las relaciones individuales que, en el marco de los flujos comerciales establecidos por los Estados conforme a normas públicas, celebran contratos con ánimo de participar en la circulación de bienes, servicios y capitales que dichos flujos generan o permiten.

Hechas estas breves consideraciones, corresponde ahora referirse en mayor detalle a la CNUCCIM, como manifestación más relevante del derecho uniforme en materia de comercio internacional, tanto desde el punto de vista jurídico como económico.

Formalmente, la CNUCCIM es un tratado internacional, que desde 1980 hasta la fecha ha sido ratificada por 85 países, siendo Brasil la última incorporación de entre los países miembros del MERCOSUR, como se verá más adelante.

Los antecedentes de la CNUCCIM se remontan incluso a 1928, en el seno del Instituto Internacional para la Unificación del Derecho Privado (UNIDROIT), que a propuesta del Profesor Ernst RABEL realizó los primeros trabajos concretos para la unificación del derecho internacional de mercaderías, iniciativa que resultaría años más tarde en las convenciones de la Haya sobre la compraventa de mercaderías, la ULIS $^{1}$ y la ULF², creadas por la Conferencia de la Haya de 1964.

Estos últimos instrumentos, aunque de escaso éxito en lo que respecta a su implementación a nivel geográfico, tuvieron como importantísimo avance la aproximación de cuatro sistemas legales -el francés, el escandinavo, el alemán y el anglosajón-y un reconocimiento práctico considerable en la jurisprudencia de varios países europeos.

En 1966, con la creación de la Comisión de las Naciones Unidas para el Derecho Mercantil Internacional (CNUDMI), la Asamblea General de la ONU reconoció que las disparidades entre las leyes nacionales que regían el comercio internacional creaban obstáculos para ese comercio, y consideró que, mediante la Comisión, la ONU podría desempeñar un papel más activo en la reducción o eliminación de esos obstáculos.

Fue así que la Asamblea General encomendó a la CNUDMI

1 Acrónimo en ingles para la Convention relating to a Uniform Law on the International Sale of Goods.

2 Acrónimo en ingles para la Convention relating to a Uniform Law on the Formation of Contracts for the International Sale of Goods. 
el trabajo de la progresiva armonización del derecho comercial internacional, cuyos proyectos debían incluir, entre otras cosas, la redacción de instrumentos aceptables a nivel mundial.

Tomando precisamente como base la ULIS y la ULF, la CNUDMI - mediante sus conferencias anuales y un Grupo de Trabajo especialmente conformado- trabajó en el proyecto y los comentarios que sirvieron de base para la Conferencia de Viena celebrada en 1980, donde fue aprobado el texto final de la CNUCCIM.

Es de notar que una importante característica de los trabajos preparatorios en el marco de la CNUDMI fue la representación balanceada de todas las regiones del mundo en la Asamblea General de la $\mathrm{CNUDMI}^{3}$, lo que a su vez aseguraba representación de los intereses tanto de los Estados industrializados con mayor desarrollo económico, como de los Estados en vías de desarrollo.

Este fue probablemente el motivo de la amplia aceptación de la CNUCCIM, evidenciado por el hecho de que los once Estados en los que la Convención originalmente entró en vigencia el 1 de enero de 1988 representaban las principales regiones geográficas, niveles de desarrollo económico y sistemas jurídicos, sociales y económicos del mundo 4 .

Más allá de la materia de la que se ocupa puntualmente, el objetivo de la CNUCCIM - sintetizado en su Preámbulo- es el de contribuir a un nuevo orden económico internacional en base a la armonía y a la igualdad, para el fomento de las relaciones amistosas entre los Estados y el desarrollo del comercio internacional mediante la supresión de los obstáculos jurídicos.

Así, ya desde su objetivo, resulta evidente que la CNUCCIM está diseñada como un cuerpo legal neutro, que no concede trato preferencial a ningún sistema jurídico o interés económico, lo cual facilitó enormemente su expansión.

En cuanto a suámbito de aplicación material, la CNUCCIM se ocupa estrictamente de la compraventa internacional de mercaderías, adoptando para su definición un criterio geográfico y un criterio sustantivo.

El primer criterio restringe su aplicación a los contratos celebrados entre partes que tienen sus establecimientos en Estados diferentes, siempre y cuando dichos Estados sean contratantes de la Convención o cuando las normas de derecho internacional prevean la aplicación de la

3 Según consta en los estatutos de la CNUDMI aprobados por la Asamblea General 2205 (XXI) del 17 de diciembre de 1966, la Asamblea estaba compuesta por siete representantes de Estados de África, cinco para los Estados de Asia, cuatro para Estados de Europa Oriental, cinco para Estados de América Latina y ocho para Estados de Europa Occidental y otros Estados. El número de representantes fue posteriormente a 36 , manteniendo el balance regional original. 4 Conforme al artículo 99 de la CNUCCIM, esta entraría en vigor el primer día del mes siguiente a la expiración de un plazo de doce meses, contados desde la fecha en que haya sido adoptado el décimo instrumento de ratificación, aceptación, aprobación o adhesión, requisito que fue satisfecho el 1 de enero de 1988. Los Estados miembros originales fueron Argentina, China, Egipto, Francia, Hungría, Italia, Lesoto, Siria, Estados Unidos, Yugoslavia y Zambia. 
ley de un Estado contratante ${ }^{5}$, excluyendo con ello de forma categórica su aplicabilidad a compraventas internas.

El segundo criterio se concentra en la determinación del alcance de este tipo de contratación, considerando compraventas los contratos de suministros de mercaderías que hayan de ser manufacturadas o producidas, a menos que la parte que las encargue asuma la obligación de proporcionar una parte sustancial de los materiales necesarios para esa manufactura o producción, y excluyendo de su aplicación a los contratos en los que la parte principal de las obligaciones de la parte que proporcione las mercaderías consista en suministrar mano de obra o prestar otros servicios ${ }^{6}$.

Las disposiciones iniciales de la CNUCCIM contemplan además una serie de compraventas que, en razón de su objeto, son excluidas de su ámbito de aplicación, i.e. contratos de consumo, compraventas judiciales y en subastas, compraventas de valores mobiliarios, títulos o efectos de comercio y dinero, compraventas de buques, embarcaciones, aerodeslizadores y aeronaves, y compraventas de electricidad ${ }^{7}$. La doctrina ha entendido que algunas de estas exclusiones se refieren a compraventas que, aunque celebradas entre sujetos con establecimientos en Estados diferentes, no tienen desde un punto de vista económico un carácter internacional. Otras exclusiones obedecen a la dificultad en la definición de la naturaleza del bien objeto del contrato. Una explicación más sencilla - y quizás la más acertada - para su exclusión es que no podía alcanzarse la unificación de las reglas para dichos contratos de compraventa.

La CNUCCIM incluye además reglas propias de interpretación, en las que se da preponderancia al carácter internacional y la necesidad de promover la uniformidad en su aplicación y de asegurar la observancia de la buena fe en el comercio internacional ${ }^{8}$, reconociendo además criterios de interpretación de los contratos en general comúnmente aceptados $^{9}$, y otorgando valor a los usos y prácticas en línea con el fenómeno del tráfico mercantil ${ }^{10}$.

Una distinción fundamental de la CNUCCIM en relación con sus antecesoras de La Haya es que reúne en un mismo cuerpo normativo la reglas aplicables a la formación del contrato y las reglas que regulan el derecho sustantivo de la compraventa, extendiendo con ello su campo de acción más allá del fenómeno de la compraventa y evitando además la existencia de normas contradictorias.

Determinadas cuestiones de la compraventa internacional de mercancías, como la validez del contrato y los efectos del contrato

5 Conforme al artículo 1.1 de la CNUCCIM.

6 Conforme al artículo 3 de la CNUCCIM.

7 Conforme al artículo 2 de la CNUCCIM.

8 Conforme al artículo 7 de la CNUCCIM.

9 Criterios enumerados en el artículo 8 de la CNUCCIM.

10 Conforme al artículo 9 de la CNUCCIM. 
sobre la propiedad de los bienes vendidos, no entran en el ámbito de la Convención. La Convención sin embargo trata de las obligaciones de las partes en el contrato, i.e. las obligaciones de los vendedores de entregar las mercancías conforme a la cantidad y la calidad estipuladas en el contrato, así como en documentos conexos, y la de transferir la propiedad de los bienes, y las obligaciones de los compradores de pagar el precio estipulado y a recibir las mercancías entregadas.

Además, la Convención prevé reglas comunes sobre las vías de recurso aplicables en caso de incumplimiento del contrato, incluyendo reglas sobre indemnización de daños y perjuicios y resolución del contrato en caso de incumplimientos sustanciales.

Entre las disposiciones suplementarias se regulan la transmisión del riesgo, el incumplimiento previo del contrato, los daños y perjuicios, y la exención del deber de cumplir el contrato.

LaCNUCCIM contempla además un sistema de reservas, destinado a excluir o modificar los efectos jurídicos de ciertas disposiciones de la Convención en su aplicación al Estado que así lo invocara. La admisión de reservas a la CNUCCIM tiene su fundamento en la voluntad de incorporar el mayor número de Estados a la Convención, difundiendo con ello su ámbito de aplicación y permitiendo que entren a formar parte Estados que no estarían dispuestos a hacerlo si no se les permitiera excluir o modificar ciertas disposiciones que estimen inaceptables.

Ahora bien, las reservas admisibles a la CNUCCIM son numerus clausus, limitándose en líneas generales a cinco: la de vinculación del Estado por solo una parte de la Convención ${ }^{11}$, la de vinculación de solo una parte del Estado por la Convención ${ }^{12}$, la de exclusión de la aplicación de la Convención a compraventas en que las partes tengan sus establecimientos en Estados empeñados en un proceso de codificación regional ${ }^{13}$ o que exijan una forma escrita para la conclusión de tales contratos ${ }^{14}$, y la subordinación de la aplicabilidad de la Convención a la reciprocidad ${ }^{15}$.

Volviendo a lo antedicho acerca de la naturaleza jurídica de la Convención, se debe tener presente que como tal, esta genera un vínculo entre los Estados regido por el derecho internacional público, y principalmente por las reglas de la Convención de Viena de 1969 sobre el derecho de los tratados para los Estados que han ratificado dicho instrumento, en virtud de la cual todo tratado en vigor obliga a las partes y debe ser cumplido por ellas de buena $\mathrm{fe}^{16}$.

Esto implica que, cumplidos los presupuestos para la aplicabilidad de la CNUCCIM arriba mencionados, sus disposiciones son -

11 Conforme al art. 92 de la CNUCCIM.

12 Conforme al art. 93 de la CNUCCIM.

13 Conforme al art. 94 de la CNUCCIM.

14 Conforme al art. 96 de la CNUCCIM.

15 Conforme al art. 95 de la CNUCCIM.

16 En virtud del art. 26 de la Convención de Viena de 1969 sobre el derecho de los tratados. 
en principio- de aplicación directa y obligatoria por los Estados contratantes a través de sus órganos jurisdiccionales, que ya no habrán de aplicarla como un derecho extranjero o internacional, sino como un derecho uniforme de su Estado. Así pues, la doctrina ha sostenido acertadamente que, en tal escenario, la aplicabilidad de la Convención no depende de una petición de parte sino que debe ser aplicada por el juez nacional ex officio cuando se cumplan los requisitos establecidos por las disposiciones antes referidas.

No obstante, esta obligatoriedad para los Estados que ratifican la Convención es relativa, por tres motivos.

El primero de ellos está contenido en la misma Convención, y se refiere a la posibilidad de las partes de un contrato de compraventa internacional de mercaderías de excluir la aplicación de la CNUCCIM o establecer excepciones a cualquiera de sus disposiciones o modificar sus efectos ${ }^{17}$. Por ende, aún reunidos los requisitos de aplicabilidad de la Convención, el intérprete de la relación contractual debe determinar si existe o no una exclusión de sus disposiciones mutuamente acordada por los contratantes. Esta exclusión puede ser expresa o tácita, aunque la doctrina ha entendido que esta última hipótesis permita identificar una voluntad real y consciente de las partes de excluir la aplicación de la CNUCCIM, limitando en la mayor medida la facultad del juez o árbitro de reconstruir a partir de indicios, o emplear presunciones para decidir cuál hubiera sido la voluntad de las partes.

El segundo motivo, aunque hipotético e improbable, guarda relación con el orden de prelación normativo de los ordenamientos jurídicos de los Estados que ratifican la Convención. Dependiendo de la jerarquía conferida a los tratados constitucionales dentro de la estructura normativa de un Estado, podría darse el caso en que una disposición de la CNUCCIM colisione y por ende ceda ante una norma de superior jerarquía.

El tercer motivo, sobre el que se pondrá énfasis en los siguientes apartados, es de índole práctica y especialmente notorio entre los países del MERCOSUR, y se refiere al escasísimo desarrollo jurisprudencial relacionado con la CNUCCIM, a pesar del intenso tráfico comercial que existe a nivel interno del bloque regional y con otros países signatarios de la Convención.

En conclusión para este punto, la CNUCCIM tiene un vasto ámbito geográfico y material de aplicación, erigiéndose como un instrumento que regula de forma integral la compraventa internacional de mercaderías a través de normas asimilables a prácticamente todo ordenamiento jurídico a nivel mundial.

$\overline{17 \text { Conforme al art. }} 6$ de la CNUCCIM. 


\section{SITUACIÓN NORMATIVA Y JURISPRUDENCIAL ACTUAL DE LA CNUCCIM EN LOS PAÍSES MIEMBROS DEL MERCOSUR}

A pesar de su reciente expansión regional con las últimas ratificaciones de Paraguay (2005) y Brasil (2014), la situación de la CNUCCIM es asimétrica entre los países del MERCOSUR.

En este sentido, se advierte que la República Bolivariana de Venezuela, a pesar de haber sido uno de los primeros países en suscribir la Convención en el año 1981, no la ha ratificado hasta la fecha. Tampoco el Estado Plurinacional de Bolivia, en inminente proceso de adhesión como miembro pleno del MERCOSUR, ha suscripto ni ratificado la Convención ${ }^{18}$.

Respecto de los demás países, Argentina fue el primero de la región - y de hecho uno de los primeros once países en el mundoen adherirse a la Convención, por ley No 22.765 promulgada el 24 de marzo de 1983, entrando esta en vigencia desde el 1 de enero de 1988.

Conforme al artículo 2 de la citada ley, Argentina formuló expresa reserva, en el sentido de que cualquier disposición del artículo 11, del artículo 29 o de la parte II de la Convención que permita que la celebración, la modificación o la extinción por mutuo acuerdo del contrato de compraventa, o la oferta, la aceptación o cualquier otra manifestación de intención, se hagan por un procedimiento que no sea por escrito, sea inaplicable en el caso de que cualquiera de las partes tenga su establecimiento en la República Argentina.

En cuanto a su orden de prelación en el ordenamiento jurídico, la Constitución de la Nación Argentina reconoce expresamente a los tratados internacionales jerarquía superior a las leyes, aunque inferior a la propia Constitución ${ }^{19}$.

En el caso de Uruguay, la CNUCCIM fue aprobada sin reservas por ley No 16.879, promulgada el 21 de octubre de 1997. La Constitución de este país, si bien hace referencia a los tratados internacionales y al derecho internacional, no dispone de forma expresa su rango o jerarquía en el ordenamiento jurídico interno ${ }^{20}$. Este silencio normativo ha sido cubierto por la jurisprudencia de la máxima instancia judicial uruguaya,

\footnotetext{
18 Según datos disponibles en el sitio web oficial de la CNUDMI: Disponible en: <http://www. uncitral.org/uncitral/es/uncitral_texts/sale_goods/1980CISG_status.html> [consultada en enero de 2017]

19 Conforme a los arts. 31 y 75 num. 22) de la Constitución de la Nación Argentina.

20 En tal sentido, el art. 6 de la Constitución Uruguaya se limita a la siguiente referencia sobre los tratados internacionales: En los tratados internacionales que celebre la República propondrá la cláusula de que todas las diferencias que surjan entre las partes contratantes, serán decididas por el arbitraje u otros medios pacíficos. La República procurará la integración social y económica de los Estados Latinoamericanos, especialmente en lo que se refiere a la defensa común de sus productos y materias primas. Asimismo, propenderá a la efectiva complementación de sus servicios públicos.
} 
que ha otorgado igual jerarquía a los tratados y a las leyes ${ }^{21}$.

Paraguay también ha aprobado la Convención, por ley No 2611 promulgada el 2 de junio de 2005, haciendo la misma reserva que Argentina bajo el artículo 96 de la Convención. Jerárquicamente, los tratados, convenios y acuerdos internacionales aprobados y ratificados solamente están por debajo de la Constitución, teniendo por tanto prelación sobre las leyes domésticas ${ }^{22}$.

Brasil fue el último país del bloque que aprobó la CNUCCIM, sin reservas, por decreto legislativo No 538 del 18 de octubre de 2012, promulgado por decreto No 8327 del 16 de octubre de 2014. Al igual que en el caso uruguayo, la Constitución de la República Federativa de Brasil no consagra de forma expresa la jerarquía normativa de los tratados internacionales incorporados al derecho positivo nacional, aunque la jurisprudencia del Supremo Tribunal Federal ha seguido la tesis de que estos tienen el mismo nivel jerárquico que las leyes domésticas, pudiendo por ende ser revocados por una ley posterior o declarados inaplicables por una ley específica ${ }^{23}$.

Ahora bien, a pesar de su vigencia en cuatro de los países del bloque regional, se observa el aún pobre desarrollo de jurisprudencia nacional relacionada con la CNUCCIM, tanto en la cantidad de fallos dictados como en la relevancia analítica de las disposiciones de la Convención. Se exponen a continuación algunos de estos fallos, advirtiendo que esta no es una lista exhaustiva, y se basa exclusivamente en los resultados de una investigación de bases de datos abiertas especializadas en la CNUCCIM y en jurisprudencia de los países miembros del MERCOSUR.

Dicho esto, se ha observado que Argentina es el país del MERCOSUR con la mayor cantidad de fallos reportados desde 1991, disponibles en la base de datos de la Pace Law Scbool ${ }^{24}$. Entre los 22 casos reportados, destacan aquellos en los que la aplicabilidad de la Convención fue objeto de análisis.

Por ejemplo, el primer caso reportado data del año 1991, e

21 Esta interpretación fue seguida por la Suprema Corte de Justicia de Uruguay, al afirmar que cabe compartir la tesis de que la ley ulterior al tratado, inconciliable con éste, supone su derogación (Suprema Corte de Justicia de Uruguay en "G. S., J. con A. del U. y C. U. S. A., Reinstalación, Casación”. Sentencia del 20 de junio de 1990. Disponible en Revista Uruguaya de Derecho Constitucional y Político, Montevideo, t. VII, núm. 37, junio-julio de 1990, p. 32-39. 22 Conforme al art. 137 de la Constitución Nacional de la República del Paraguay.

23 En este sentido se ha pronunciado el Supremo Tribunal Federal respecto de la aplicabilidad de una convención internacional y su relación con las leyes del país, afirmando que aunque la Convención de Ginebra que previó una ley uniforme sobre letras de cambio y pagarés tenga aplicabilidad en el derecho interno brasileño, esta no se superpone a las leyes del país, de ahí la constitucionalidad y consecuente validez del Decreto Ley $n^{\circ} 427 / 69$, que instituyó el registro obligatorio de la Nota Promissória em Repartição Fazendária, bajo pena de nulidad del título (Supremo Tribunal Federal - RE: 80004 SE, Relator: Min. Xavier de Albuquerque, decisión de fecha 01 de junio de 1977, Fecha de publicación: DJ 29-12-1977).

24 Disponible en: <http://www.cisg.law.pace.edu/cisg/text/casecit.html\#argentina $>$ [consultada en enero de 2017]. 
involucra a una empresa argentina y una empresa belga en el marco de un contrato de compraventa de maquinaria ${ }^{25}$. En este caso, la Cámara de Apelaciones entró al estudio de aplicación de la Convención sobre la base de la cláusula de derecho transitorio contenida en el artículo 100 , declarando su inaplicabilidad en atención a que el objeto del litigio databa del año 1970, es decir, antes a la fecha en que la Convención quedase abierta a la firma de los Estados.

En el caso Mayer, Alejandro c. Onda Hofferle Gmbh \& C. s/ ordinario $^{26}$, referente a un supuesto incumplimiento de un contrato de compraventa de carbón vegetal celebrado entre una empresa situada en Argentina y otra situada en Alemania, la Cámara Nacional de Apelaciones en lo Comercial de Buenos Aires determinó que la relación contractual no se encuadraba dentro del supuesto de aplicación previsto en el art. 1.1 a) de la Convención, pues si bien esta se hallaba en vigor en Argentina desde el 1 de enero de 1988, año en que las partes perfeccionaron el contrato, no entró en vigor en Alemania sino hasta el 1 de enero de 1994. Sin embargo, el órgano jurisdiccional entendió que la Convención sí era aplicable bajo el supuesto del artículo 1.1.b), en base a las normas del derecho internacional privado argentino vigentes, que establecían que la ley aplicable a un contrato es la del lugar de su cumplimiento. Tomando en consideración que la prestación más característica del contrato de compraventa - la entrega de la cosa- y que el contrato objeto de la litis tenía cláusula FOB Buenos Aires, el tribunal concluyó que, al designar las normas de derecho internacional privado la aplicación del derecho argentino, y al ser Argentina un Estado que había ratificado la Convención, cabía su aplicación en virtud del artículo 1.1.b).

Más recientemente, la Cámara Nacional de Apelaciones en lo Comercial de Buenos Aires emitió su decisión en el caso Ecotune Private Ltd. c/ Cencosud S.A / ordinario ${ }^{27}$, que involucraba a una empresa argentina y una empresa india en una contienda relacionada con una compraventa internacional de mercaderías. El tribunal entendió, en grado de apelación, que a pesar de que el Estado de una de las partes contratantes - India - no es parte de la Convención, esta es aplicable sobre la base del artículo 1.1.b), puesto que las normas de derecho

25 Cámara Nacional de Apelaciones en lo Comercial de Buenos Aires, Sala C, en Quilmes Combustibles S.A.C.I.c. Vigan, S.A., sentencia del 15 de marzo de 1991. Texto completo disponible en: <http://www.cisgspanish.com/jurisprudencia/argentina/15-marzo-1991-camara-nacionalde-apelaciones-en-lo-comercial-de-buenos-aires/> [consultada en enero de 2017].

26 Cámara Nacional de Apelaciones en lo Comercial de Buenos Aires, Sala E, en Mayer, Alejandro c. Onda Hofferle Gmbh \& C. S/ordinario, sentencia del 24 de abril de 2000. Texto completo disponible en: <http://www.cisgspanish.com/jurisprudencia/argentina/camaranacional-de-apelaciones-en-lo-comercial-24-abril-2000/> [consultada en enero de 2017].

27 Cámara Nacional de Apelaciones en lo Comercial de Buenos Aires, Sala F, en Ecotune (India) Private Ltd. c/ Cencosud S.A. s/ ordinario, sentencia del 7 de octubre de 2010. Texto completo disponible en: <http://www.cisgspanish.com/jurisprudencia/argentina/camaranacional-de-apelaciones-en-lo-comercial-de-buenos-aires-sala-f-7-de-octubre-de-2010/> [consultada en enero de 2017]. 
internacional privado del foro - Argentina - llevaban a la aplicación del derecho argentino, como ley del lugar de cumplimiento del contrato.

Brasil sigue en la cantidad de fallos reportados en bases de datos especializadas, con un total de 5 sentencias disponibles en la base de datos de la Pace Law School ${ }^{28}$. Una particularidad de la jurisprudencia brasileña reportada es que comprende fallos dictados con anterioridad al año 2014, es decir, antes de que la CNUCCIM entrara en vigencia en Brasil, puesto que las cortes estaban dispuestas a aplicar artículos de la Convención que se consideraban consistentes con la legislación doméstica.

Este fue el caso, por ejemplo, en Prakasa Indústria e Comércio de Utilidades do Lar Ltda. c. Mercomáquinas Indústria, Comércio e Representações $L t d a^{29}$, que involucraba a dos contratantes brasileños, y en el que el tribunal nacional hizo referencias a observaciones obiter dicta a los artículos 8.3) y 77 de la Convención, en relación con las obligaciones de una parte que invoca el incumplimiento de contrato para reducir las pérdidas derivadas de dicho incumplimiento, y sobre los principios de equidad y buena fe en la ejecución y el cumplimiento de los contratos; todo ello, en atención a la compatibilidad de estas disposiciones con la Reformulación de las normas jurisprudenciales y la legislación civil brasileña.

En el caso José HenriqueS. N. de Souza y otroc. Construtora Costa Norte Empreendimentos Imobiliários SC Ltda. ${ }^{30}$, el tribunal brasileño se refirió nuevamente obiter dicta a la Convención, en un litigio entre contratantes brasileños que versaba principalmente sobre la doctrina de la exceptio de non adimpleti contractus, consagrada en el artículo 72 de la Convención, y que reconoce que toda parte que incurra en un incumplimiento esencial del contrato no podrá obligar a la otra parte a que cumpla con las obligaciones que hubiera contraído en virtud de dicho contrato.

Aunque con menor frecuencia y en casos aislados, la jurisprudencia uruguaya también ha invocado las disposiciones de la CNUCCIM. Puntualmente, en J. JE Marketing Distribution Center Inc. c/ CABA S.A. y otros s/ daños y perjuicios ${ }^{31}$, el Tribunal de Apelación en lo Civil determinó la aplicabilidad de la Convención a un contrato

28 Disponible en: < http://www.cisg.law.pace.edu/cisg/text/casecit.html\#brazil > [consultada en enero de 2017].

29 Tribunal de Apelación del Estado de Rio Grande do Sul, Sala 5a de lo civil, en Prakasa Indústria e Comércio de Utilidades do Lar Ltda. c. Mercomáquinas Indústria, Comércio e Representações Ltda., sentencia del 20 de mayo de 2009. Resumen del fallo en español disponible en: <https://documents-dds-ny.un.org/doc/UNDOC/GEN/V12/556/54/PDF/ V1255654.pdf?OpenElement> [consultada en enero de 2017].

30 Tribunal de Apelación del Estado de São Paulo - Sala Cuarta de Derecho Privado, en José Henrique S. N. de Souza y otro c. Construtora Costa Norte Empreendimentos Imobiliários SC Ltda., sentencia del 24 de abril de 2008. Resumen del fallo en español disponible en: $<$ https://documents-dds-ny.un.org/doc/UNDOC/GEN/V12/556/54/PDF/V1255654. pdf?OpenElement> [consultada en enero de 2017].

31 Tribunal de Apelaciones en lo Civil del Séptimo Turno en J. JE Marketing Distribution Center Inc. c/ CABA S.A. y otros s/ daños y perjuicios, sentencia del 13 de mayo de 2010. Texto completo disponible en: <http://bjn.poderjudicial.gub.uy $>$ [consultada en enero de 2017]. 
celebrado entre una empresa estadounidense y una uruguaya, para la determinación del concepto de "plazo razonable" para la verificación de mercaderías, en forma armónica con los propios términos del artículo 7.2 de la Convención.

En otro caso, RTM Grupo Inc. c/ OFEROL S.A. y otros s/ rescisión de contrato ${ }^{32}$, el tribunal de alzada hizo referencia a los artículos 3 y 29 de la Convención para determinar la naturaleza de la relación entre demandante y demandado, y específicamente la ausencia de legitimación pasiva causal en la litis.

Finalmente, debe señalarse la casi nula mención a la CNUCCIM en la jurisprudencia paraguaya. En efecto, a pesar de su adhesión ya en el 2005, solamente existe un fallo reportado, en el caso Alex S.A. c/ Seguros Chaco S.A. de Seguros y Reaseguros s/ cumplimiento de contrato $y$ cobro de guaraníes ${ }^{33}$, en el que la Corte Suprema de Justicia refirió obiter dictum la Convención para fundar su decisión en relación con el traspaso del riesgo por la pérdida o destrucción durante el transporte de mercaderías, aunque se hizo la expresa salvedad de que la mención es exempli gratia, puesto que la Convención aún no se hallaba vigente a la fecha de la operación objeto de la litis.

La CNUCCIM también ha sido incorporada a los ordenamientos jurídicos de la mayoría de los Estados Asociados al MERCOSUR, siendo Surinam la única excepción ${ }^{34}$. A eso hay que agregar que la amplia lista de países miembros incluye a los Estados Unidos, China, Rusia y la Unión Europea, todos mercados claves para la economía del bloque regional y con una rica jurisprudencia de casos relacionados con la Convención.

\section{4. ¿CUÁLES SON LOS MOTIVOS PARA LA ESCASA JURISPRUDENCIA RELACIONADA CON LA CNUCCIM EN EL MERCOSUR?}

Lo expuesto en el apartado anterior demuestra que la CNUCCIM no es solamente una convención incorporada en la mayoría de los

32 Tribunal de Apelaciones en lo Civil del Cuarto Turno, en RTM Grupo Inc. c/ OFEROL S.A. y otros s/ rescisión de contrato, sentencia del 27 de noviembre de 2013. Texto completo disponible en: <http://bjn.poderjudicial.gub.uy> [consultada en enero de 2017].

33 Corte Suprema de Justicia, Sala Civil, en Alex S.A. c/ Seguros Chaco S.A. de Seguros y Reaseguros s/ cumplimiento de contrato y cobro de guaraníes, sentencia del 16 de abril de 2009 . Texto completo disponible en: <http://www.csj.gov.py/jurisprudencia/> [consultada en enero de 2017].

34 En Chile, la CNUCCIM está en vigencia desde el 1 de marzo de 1991, con la misma reserva formulada sobre la base del artículo 96 que Argentina y Paraguay. En Ecuador, la Convención está en vigencia desde el 1 de febrero de 1993, sin reservas. En Perú, la Convención está en vigencia sin reservas desde el 1 de abril de 2000. Colombia adoptó la CNUCCIM está en vigencia, sin reservas, desde el 1 de agosto de 2002. Finalmente en Guyana, la Convención entró en vigencia desde el 1 de octubre de 2015. Datos disponibles en la base de datos de la Pace Law School: <http://www.cisg.law.pace.edu/cisg/countries/cntries.html> [consultada en enero de 2017]. 
Estados Partes y Asociados del MERCOSUR, sino que además es un instrumento estratégico para el fortalecimiento de las relaciones comerciales tanto entre los países del bloque como entre estos y sus principales mercados internacionales.

A pesar de todo ello, y como también se ha visto, la utilización de la CNUCCIM en la jurisprudencia regional ha sido escasísima y, salvo dignas excepciones, mayoritariamente intrascendente en el escenario global.

Esto nos lleva indefectiblemente a preguntarnos cuáles podrían ser las razones que expliquen esta carencia. Para ello, resulta especialmente ilustrativa la metodología seguida por el Dr. Juan Antonio Gaviria-Gil en un estudio enfocado específicamente en la ausencia de casos sobre la CNUCCIM en Colombia.

Corresponde advertir preliminarmente que muchas de las hipotéticas causas escapan de toda medición, como la posibilidad de que los comerciantes internacionales que contratan bajo las reglas de la CNUCCIM tiendan en la mayoría de sus transacciones a cumplir con sus obligaciones contractuales, o en caso de incumplimiento prefieran la aplicación de sanciones extra-jurisdiccionales, excluyendo en ambos casos la opción de recurrir a órganos jurisdiccionales para la solución de controversias.

También es posible que, en los casos en los que esté contemplada la CNUCCIM como derecho sustancial, los contratantes prefieran someter las eventuales controversias a tribunales arbitrales con sede en alguno de los países del MERCOSUR, pero con pacto de confidencialidad de los procedimientos. Esta alternativa no obstante es improbable, habida cuenta del aún incipiente uso del arbitraje como mecanismo de solución de controversias en los países integrantes del MERCOSUR, tema que escapa del objeto de este trabajo.

Hechas estas acotaciones, se pasa a continuación al estudio de cuatro hipótesis que podrían explicar la escasa jurisprudencia regional: la escasa relevancia del comercio internacional para el MERCOSUR; la expresa exclusión de la CNUCCIM como ley sustancial de los contratos de compraventas con contratantes del MERCOSUR; (III) la aplicabilidad de la CNUCCIM como ley sustancial de los contratos, pero la estipulación de una jurisdicción de otro país o tribunal arbitral con sede fuera de los países del MERCOSUR; y (IV) la falta de conocimiento sobre la CNUCCIM.

\subsection{Escasa relevancia del comercio internacional para el MERCOSUR}

En el informe MERCOSUR No 21 del Banco Interamericano de Desarrollo (BID), correspondiente al segundo semestre de 2015 y al 
primer semestre de 2016, se pone en resalto que el comercio del bloque regional se contrajo en el periodo observado, siendo este desempeño negativo en todos los países del bloque, con una profundización de la tendencia a la baja en las exportaciones registrada desde el 2012, y una acentuación en la caída de las importaciones, que se presenta desde el 2014.

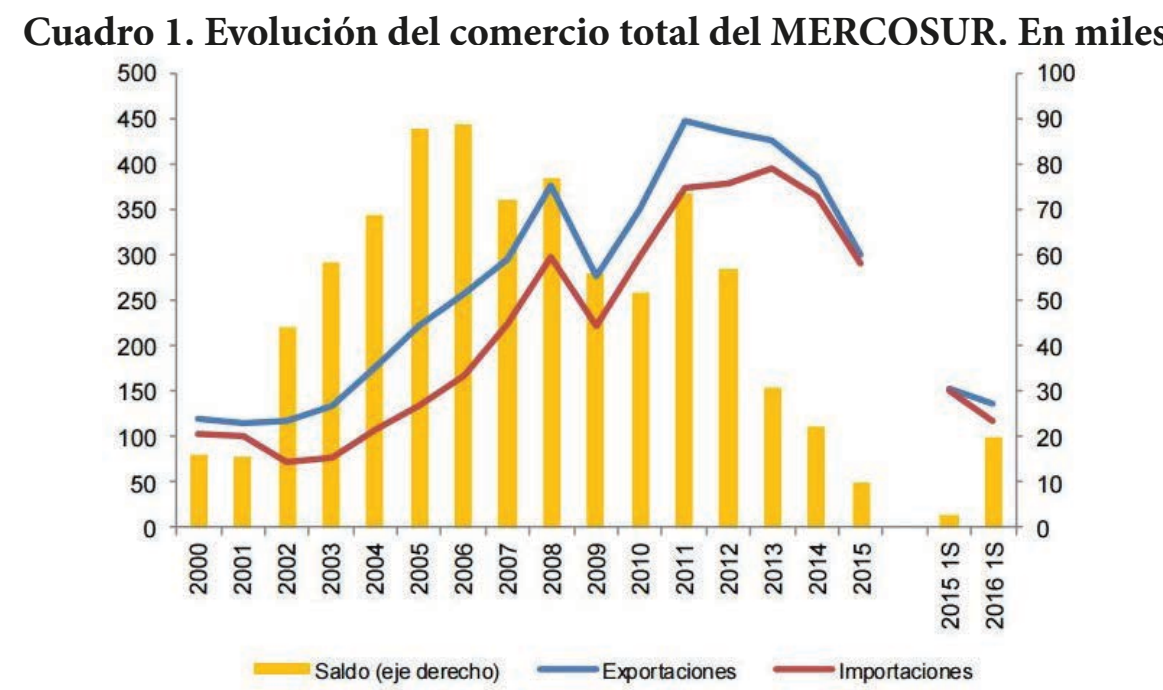

Fuente: Banco Interamericano de Desarrollo - Informe MERCOSUR No 21

Esta tendencia fue además generalizada en los cinco países miembros del bloque regional, incluyendo a Bolivia.

Cuadro 2. Comercio del MERCOSUR por país. Valor en millones de US\$ y variación interanual en porcentaje

\begin{tabular}{|c|c|c|c|c|c|c|c|c|}
\hline \multirow{3}{*}{ Pais } & \multicolumn{4}{|c|}{ Exportaciones } & \multicolumn{4}{|c|}{ Importaciones } \\
\hline & \multicolumn{2}{|c|}{2015} & \multicolumn{2}{|c|}{ 1Sem. 2016} & \multicolumn{2}{|c|}{2015} & \multicolumn{2}{|c|}{$1^{\circ} \mathrm{Sem} .2016$} \\
\hline & Valor & Var. i.a. & Valor & Var. i.a. & Valor & Var. i.a. & Valor & Var. i.a. \\
\hline Argentina & 56.752 & $-17,0$ & 27.741 & $-2,5$ & 59.787 & $-8,3$ & 27.256 & $-5,8$ \\
\hline Brasil & 191.134 & $-15,1$ & 90.253 & $-4,3$ & 171.449 & $-25,2$ & 66.601 & $-27,7$ \\
\hline Paraguay & 8.357 & $-13,3$ & 4.475 & $-1,4$ & 9.529 & $-15,7$ & 3.955 & $-18,0$ \\
\hline Uruguay & 7.685 & $-15,8$ & 3.367 & $-15,4$ & 9.489 & $-17,4$ & 3.934 & $-22,3$ \\
\hline Venezuela & 36.374 & $-51,3$ & 10.873 & $-48,3$ & 40.100 & $-15,6$ & 14.939 & $-20,2$ \\
\hline MERCOSUR & 300.302 & $-22,4$ & 136.709 & $-10,3$ & 290.355 & $-20,4$ & 116.685 & $-22,0$ \\
\hline Bolivia & 8.726 & $-32,4$ & 3.324 & $-27,9$ & 9.119 & $-8,5$ & 3.741 & $-14,4$ \\
\hline
\end{tabular}

Fuente: Banco Interamericano de Desarrollo - Informe MERCOSUR No 21

Estas cifras, aunque elocuentes en lo que respecta a la desaceleración que está experimentando el bloque, son insuficientes para concluir que el comercio internacional $-\mathrm{y}$ por ende la eventual utilidad de la 
CNUCCIM - carecen de relevancia para el MERCOSUR.

En efecto, el mismo informe expresa que, a pesar de la reducción generalizada del intercambio con mercados extra-regionales en el periodo de observación, este representa más del $86 \%$ de los flujos totales del MERCOSUR, indicando que a pesar de la disminución del 22,6\% de las exportaciones extra-regionales en relación con el 2014, estas totalizaron la suma de US\$259.477 millones en el 2015.

Otro dato en el que se pone énfasis es el impacto de la desaceleración de China en las exportaciones de los países del MERCOSUR, lo que demuestra el papel clave del país asiático en el desempeño comercial del bloque regional. Este no es un dato menor, teniendo en cuenta que China -Estado miembro original de la Convención desde 1988- es el segundo país en el mundo con mayor cantidad de casos reportados en los que se ha aplicado la CNUCCIM, según la base de datos de la Pace Law School ${ }^{35}$.

Las importaciones extrazona, aunque también experimentaron una reducción del $20,1 \%$ en relación con el año anterior, totalizaron US\$ 251.832 millones en el año 2015, fenómeno que se explica en cierta medida por la disminución de los precios y por la menor demanda interna.

Otro dato relevante constituye el comercio intra-MERCOSUR, en el que las exportaciones totalizaron US\$ 40.825 millones, variando no obstante de forma sustancial la importancia relativa del mercado regional entre los miembros, con una mayor participación en los países de menor tamaño. En este sentido, los datos de 2015 reflejan que el MERCOSUR concentra más del $40 \%$ del comercio de Paraguay, cerca de $30 \%$ del de Uruguay y casi un cuarto del de Argentina, al tiempo que no alcanza el $10 \%$ del de Brasil. Estas cifras son similares tanto para las exportaciones como para las importaciones de los miembros fundadores del MERCOSUR (todos partes de la CNUCCIM a la fecha), mientras en el caso de Venezuela existe una brecha significativa entre una y otra: $11,5 \%$ de sus compras externas provienen del bloque y solo destina $2,2 \%$ de sus ventas.

Un último dato que merece atención es el que refiere a los principales productos exportados por los países del MERCOSUR, entre los que destacan la carne bovina, el trigo, la soja, los productos minerales y el petróleo, los cereales en general, los automóviles y varios otros productos que se encuentran, en principio, bajo el ámbito de aplicación de la CNUCCIM.

En resumidas cuentas, la hipótesis de que el comercio internacional - intra y extra-región- carece de relevancia para los países que integran el MERCOSUR, y por ende la CNUCCIM tiene poca utilidad práctica, es sencillamente inadmisible.

35 China tiene 432 casos reportados, solamente superado por Alemania, con 534 casos. Datos disponibles en la base de datos de la Pace Law School: <http://www.cisg.law.pace.edu/cisg/ countries/cntries.html> [consultada en enero de 2017]. 


\subsection{La expresa exclusión de la CNUCCIM como ley sustancial en los contratos de compraventa internacional de mercaderías con contratantes del MERCOSUR}

Ciertamente puede ser el caso que los compradores y vendedores opten por una ley más favorable que la CNUCCIM, ya sea como resultado de un consenso o de un mayor poder de negociación de uno de los contratantes, que exija la exclusión total o parcial de la Convención, como lo admite el artículo 6 referido supra.

Ahora bien, esta hipótesis tendría sentido si, por ejemplo, los países miembros del MERCOSUR o los contratantes de sus principales socios comerciales encontrasen inconveniente la aplicación de la CNUCCIM y prefiriesen en consecuencia recurrir a una legislación local que les ofrezca mayor neutralidad y seguridad jurídica.

Este difícilmente sea el caso, teniendo en cuenta que los principales socios comerciales de los países del MERCOSUR - China, Estados Unidos, Rusia y los países de la Unión Europea- son miembros de la Convención y cuentan con un amplio desarrollo doctrinario y jurisprudencial en la materia. No obstante, algunos estudios de campo realizados por expertos demuestran que no es inusual la exclusión expresa y rutinaria de la CNUCCIM por parte de abogados que negocian y redactan contratos para sus clientes. Entre estos, destacan los resultados obtenidos por la Prof. Ingeborg Schwenzer y el Prof. Christopher Kee, en una encuesta llevada a cabo entre 640 participantes de 85 países, que demuestra que de los abogados de países miembros de la CNUCCIM que respondieron la encuesta, 13\% manifestó excluir siempre la Convención, y otro 32\% manifestó hacerlo a veces; en contraste, $55 \%$ manifestó hacerlo rara vez o nunca.

El mismo estudio arrojó un resultado revelador: si bien muchos de los encuestados que manifestaron excluir la CNUCCIM por preferir la legislación doméstica, en el caso de América Latina el principal motivo manifestado para la exclusión es - paradójicamente-la preocupación acerca de la falta de jurisprudencia autóctona sobre la materia.

Por otra parte, y como se ha visto en la hipótesis anterior, muchos de los principales productos exportados por el MERCOSUR se venden a granel y tienen precios fluctuantes en los mercados internacionales. Para esta clase de contratación se ha sostenido que la exclusión dela CNUCCIM es relativamente frecuente, teniendo en cuenta que, aparentemente, la Convención fue redactada pensando en contratos cuyo objeto es la venta de un bien por parte de un fabricante o de un comercializador a un comprador que lo requiere para su proceso de producción o distribución, y no en negocios jurídicos con propósito especulativo, en el que los compradores no actúan usualmente como destinatarios finales, y las partes prefieren tener mayor libertad para la rescisión de los contratos 
independientemente de la relevancia del incumplimiento.

Estas hipótesis son válidas, aunque claro está que ninguna puede ser tenida por concluyente, habida cuenta del gran universo de investigación, por lo que para confirmar estas teorías se requeriría un estudio empírico y focalizado en las particularidades jurídicas y comerciales de los países del MERCOSUR.

\subsubsection{La aplicabilidad de la CNUCCIM como ley sustancial de los contratos, pero la estipulación de una jurisdicción de otro país o tribunal arbitral con sede fuera de los países del MERCOSUR}

Desde otra perspectiva, podría ser el caso que la CNUCCIM es la ley aplicable a compraventas internacionales con contratantes de los países del MERCOSUR, pero que exista una cláusula de jurisdicción que somete las eventuales controversias a jurisdicciones - ordinarias o arbitrales - con sede en otro países, hipótesis que gana mayor fuerza en la jurisdicción arbitral, siendo que los países del bloque regional son en muy raras ocasiones seleccionados como sedes de arbitraje, por su incipiente desarrollo en este mecanismo de solución de controversias.

Esta hipótesis explicaría parcialmente la limitada jurisprudencia a nivel regional, aunque exigiría una revisión pormenorizada de las bases de datos especializadas para determinar, si en casos resueltos por jueces o árbitros con sede en otros países, han intervenido contratantes de los países del MERCOSUR como demandantes o demandados, de modo a descartar la incidencia de otros factores.

\subsubsection{La falta de conocimiento sobre la CNUCCIM}

Finalmente, aunque no por ello menos factible, es la posibilidad más simple: la falta de conocimiento de la CNUCCIM por parte de los comerciantes, abogados, jueces y árbitros del MERCOSUR.

Al referirnos a la falta de conocimiento, lo hacemos en sentido amplio, tanto en lo que respecta a la existencia misma de la Convención, como a su ámbito de aplicación y sus ventajas para el comercio transfronterizo.

Una realidad innegable en el MERCOSUR, a la que ya se hizo referencia en este trabajo, es la asimetría en lo que hace al tiempo de adhesión y al desarrollo jurisprudencial en materia de la Convención, que demuestra que en algunos países este instrumento de derecho uniforme es más conocido - y por ende utilizado- que en otros. Esto sin embargo no logra explicar - al menos de forma aislada- por qué Uruguay (parte desde hace 20 años) y Paraguay (parte desde hace 12 años) tienen tan poca, o de hecho nula jurisprudencia referente a la Convención, o por qué la jurisprudencia argentina, a pesar de ser 
Estado parte original de la Convención (desde hace ya casi treinta años), ha tenido relativa trascendencia a nivel mundial.

Tampoco puede obviarse el hecho probado supra de que existe una activa participación del MERCOSUR en el comercio internacional. Muchas de las empresas del bloque son multinacionales que comercializan con otras grandes empresas extranjeras y que cuentan con personal altamente calificado y abogados de primer nivel, conocedores - al menos en teoría - de la existencia de la Convención.

En relación con lo anterior, se deben mencionar los esfuerzos académicos realizados a nivel regional y mundial para la promoción de la Convención, entre los que destacan las competencias de arbitraje comercial internacional como la Willem C. Vis International Commercial Arbitration Moot, competencia anual con 24 ediciones - al año 2017que reúne a más de 300 universidades de todo el mundo, y que toma a la CNUCCIM en cada edición como ley sustancial para el caso de estudio. En esta y otras competencias, los equipos de estudiantes de universidades del MERCOSUR — algunos ya hoy jóvenes abogadoshan tenido destacadas participaciones.

Por otra parte, y aun suponiendo que fuese admisible la justificación de la falta de conocimiento de la Convención, lo cierto es que la misma constituye la ley sustancial que rige por defecto las compraventas internacionales dentro de su ámbito de aplicación, siendo necesario el pacto expreso de los contratantes para su exclusión, por lo que la mera ignorancia causal jurídica no resiste como fundamento para su falta de aplicación jurisprudencial, máxime a la luz del ampliamente aceptado principio procesal del iura novit curia, que descarta incluso la necesidad de probar su aplicabilidad, al ser ley doméstica en cuatro de los países del MERCOSUR.

\section{CONCLUSIÓN}

En este breve trabajo se ha intentado demostrar la utilidad que podría tener un instrumento de derecho uniforme como la CNUCCIM para el fortalecimiento del comercio transfronterizo de los países del MERCOSUR, promoviendo con ello los objetivos primordiales del bloque regional.

Las asimetrías en la adopción y aplicación de la Convención entre los países del bloque ciertamente no son menores, y requieren de voluntad política para nivelar las reglas de juego y llegar a una verdadera uniformidad del derecho mercosureño en la materia regulada por la CNUCCIM. Sin embargo, las ventajas que traería la adopción de reglas comunes para un acto jurídico tan elemental como la compraventa internacional de mercaderías, a través de una normativa moderna y equitativa como la Convención, justifican sobradamente todo esfuerzo en tal sentido. 
Es imperioso además que la normativa ya adoptada en la mayoría de los países del bloque sea conocida y aplicada por comerciantes y profesionales del derecho, y que en su aplicación se puedan reflejar las particularidades de los sistemas jurídicos que integran el MERCOSUR. De lo contrario, la adopción de la Convención no representa otra cosa más que un mensaje vano a la comunidad internacional, una moda pasajera que no incide - o mejor dicho, no se permite que incida - en la cultura jurídica de nuestros países.

Por de pronto, se espera que esta contribución sirva de base para identificar los verdaderos motivos para la escasa jurisprudencia autóctona en materia de la CNUCCIM, advirtiendo que las hipótesis aquí expuestas no van más allá de eso, y que un estudio mucho más detallado es necesario para un diagnóstico certero de las causas de tal escasez, así como de la conveniencia y los medios adecuados para promover una mayor utilización de la Convención en el comercio internacional de los países del MERCOSUR.

\section{AGRADECIMIENTO}

Mi sincero agradecimiento a mi colega y amiga Kyra Thielmann por sus valiosas contribuciones a este trabajo.

\section{RESUMEN BIOGRÁFICO}

Pablo Antonio Debuchy Boselli es Abogado, egresado de la Universidad Católica "Nuestra Señora de la Asunción”, de Asunción, Paraguay, 2010. Master of Laws in International Legal Practice del Instituto de Empresa, Madrid, España, Año 2012. Actualmente es Asociado del Estudio Jurídico Gross Brown de Asunción, Paraguay.

\section{REFERENCIAS BIBLIOGRÁFICAS}

BANCO INTERAMERICANO DE DESARROLLO. INSTITUTO PARA LA INTEGRACIÓN DE AMÉRICA LATINA Y EL CARIBE. Informe MERCOSUR No 21/ BID-INTAL - Segundo semestre 2015 - Primer semestre 2016. 2016, [consultado en enero de 2017]. Disponible en <https:// publications.iadb.org/bitstream/handle/11319/7959/Informe-MERCOSURNo-21-2015-2016-Segundo-semestre-2015-Primer-semestre-2016.pdf>

BASEDOW, Jürgen; FERNANDEZ ARROYO, Diego P.; MORENO RODRIGUEZ, José A (eds.). ¿Cómo se codifica hoy el derecho comercial internacional? Asunción: La Ley, 2010.

DIEZ-PICAZO, Luis; DIEZ PICAZO y PONCE DE LEON, Luis María; CABANILLAS SÁNCHEZ, Antonio. La compraventa internacional de mercaderías: Comentario a la Convención de Viena. Cizur Menor 
(Navarra): Editorial Aranzadi, 1998.

FELEMEGAS, John. The United Nations Convention on Contracts for the International Sale of Goods, en Pace Review of the Convention on Contracts for the International Sale of Goods (CISG), 2000, p. 115-265 [consultado en enero de 2017]. Disponible en: <http://www.cisg.law. pace.edu/cisg/biblio/felemegas.html\#N_1>

FERNANDEZ ARROYO, Diego P. (ed.). Derecho internacional privado de los Estados del MERCOSUR. Buenos Aires: Zavalía, 2003.

FERRARI, Franco (2009). "The CISG and its Impact on National Legal Systems". The Modern Law Review. 2009, vol 72, nº 5, p. 867-873.

GARRO, Alejandro. "Unification and Harmonization of Private International Law in Latin America. American Journal of Comparative Law. 1992, n 40, p. 587-616 [consultado en enero de 2017]. Disponible en: $<$ http://www.cisg.law.pace.edu/cisg/biblio/garro3.html\#>

GAVIRIA-GIL, Juan Antonio. "El enigma de la ausencia de casos sobre CISG en Colombia". International Law, Revista Colombiana de Derecho Internacional. 2015, $\mathrm{n}^{\circ} 26$, p. 249-288 [consultado en enero de 2017]. Disponible en: $<$ http://dx.doi.org/10.11144/Javeriana.ill5-26.eacc $>$

ILLESCAS ORTIZ, Rafael; PERALES VISCASILLAS, María del Pilar. Derecho mercantil internacional. El derecho uniforme. Madrid: Editorial Universitaria Ramón Areces, 2003.

PIZZOLO, Calogero. Derecho e integración regional: Comunidad Andina, Mercosur, SICA, Unión Europea. Buenos Aires: Ediar, 2010.

RUIZ DIAZ LABRANO, Roberto. Derecho Internacional Privado. Asunción: La Ley Paraguaya, 2010.

SCHWENZER, Ingeborg y KEE, Christopher. "Global Sales Law Theory en Practice". En SCHWENZER Ingeborg y SPAGNOLO Lisa (eds.). Toward Uniformity: the $2^{\text {nd }}$ Annual MAA Schlechtriem CISG Conference. La Haya: Eleven International Publishing, 2011.

SCHWENZER, Ingeborg, MUÑOZ, Edgardo. Schlechtreim \& Schwenzer: Comentario sobre la Convención de las Naciones Unidas sobre los Contratos de Compraventa Internacional de Mercaderías. Cizur Menor (Navarra): Editorial Aranzadi, 2011. 